\title{
Content based Image Retrieval using Selective Region Matching with Region of Interest and SVM
}

\author{
Sahil Charaya \\ M.tech Student \\ SBSSTC, Ferozepur
}

\author{
Sonika Jindal \\ Assistant Professor \\ SBSSTC, Ferozepur
}

\author{
Bhavneet Kaur \\ Assistant Professor \\ SBSSTC, Ferozepur
}

\begin{abstract}
Recently, very large collections of images and videos have grown rapidly. In parallel with this growth, content-based retrieval and querying the indexed collections are required to access visual information. Three main components of the visual information are color, texture and shape. In this paper, a selective region based content-based image retrieval system is presented that combines two visual descriptors of images and measures similarity of images by applying a SVM Classification. Paper. Here, the selective region matching with Region of Interest and SVM classification based CBIR retrieval system for imaging is presented in order to provide better image classification and fast image retrieval. In the proposed method the color and texture features like contrast, mean, standard deviation, energy and entropy are extracted from the image. Also it is shown through experimental results and analysis of retrieval effectiveness of querying that the content-based retrieval system is effective in terms of retrieval and scalability.
\end{abstract}

\section{Keywords}

Content-Based Image Retrieval (CBIR), Support Vector Machine (SVM), Color Moments, Texture.

\section{INTRODUCTION}

Content Based Image Retrieval (CBIR) is browsing, searching and navigation of images from large image databases based on their visual contents. CBIR has been an active area of research for more than a decade. Many CBIR systems have been developed; like QBIC, Simplicity, and Blob world Traditional CBIR systems use low level features like color, texture, shape and spatial location of objects to index and retrieve images from databases. Low level features can be global or local (region based) [1].Global feature based CBIR fails to compare the regions or objects in which a user may be interested. Therefore Region Based Image Retrieval (RBIR) is more effective in reflecting the user requirement. The goal of CBIR systems is to operate on image data and, in response to a visual query, extract relevant images from the dataset. The process is shown in figure 1. After a decade of intensive research, CBIR technology is now beginning to move out of the laboratory and into the marketplace, in the form of commercial products like QBIC etc. The architecture of CBIR is shown in figure 1.

A typical query image consists of both relevant and irrelevant regions. The irrelevant regions limit the effectiveness of existing content based image retrieval systems. Irrelevant regions can be removed by defining ROI in the query image [1] [3].

Depending upon the method of formulating region based query, RBIR can be categorized into two classes:

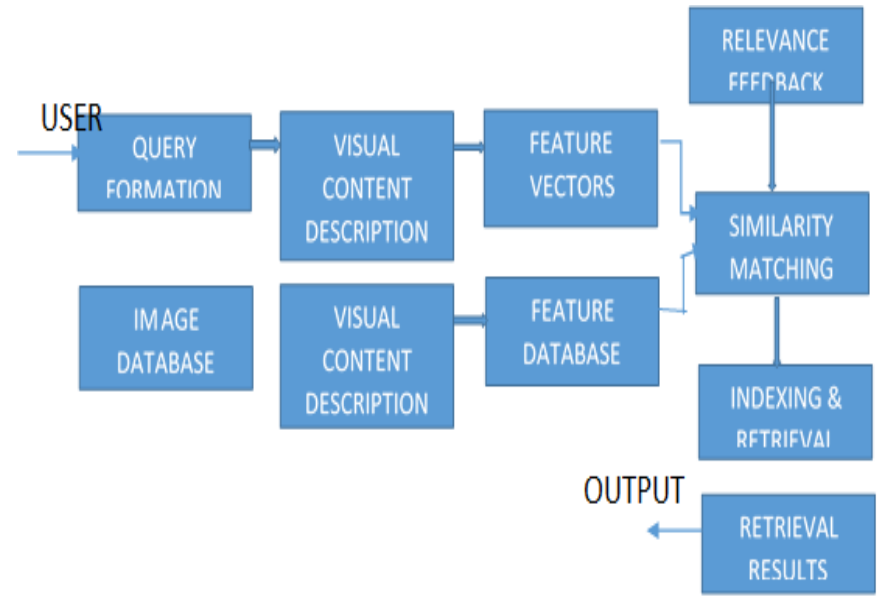

Figure 1: Architecture of CBIR

1. System Designate ROI (SDR) and

2. User Designated ROI (UDR).

These systems have various ways of querying and representing image in the database. UDR approach is more promising as it enables the user to express his intent in the query formulation. But in UDR approaches, it is difficult to formulate an accurate query if there are variations in the sizes of ROI.

\section{FEATURE EXTRACTION}

The feature extraction finds the image detail in the form of feature value (or a set of value called a feature vector) for each pixel. Region based retrieval systems generally use small feature vector because if the number of regions in the layout is high then it takes a lot of time to compare features of all regions within the query image. Proposed approach requires selective comparisons; hence the length of feature vector can be increased to represent the region accurately without affecting the computation time. In the proposed technique, HSV has been used for color and GLCM for texture features [10] [12].

\subsection{Image Retrieval using Colour Content}

Colour is very easily available image feature, which generally is represented in form of colour components of digital image [14].The color feature has widely been used in CBIR systems, because of its easy and fast computation. Color is also an intuitive feature and plays an important role in image matching. The extraction of color features from digital images depends on an understanding of the theory of color and the representation of color in digital images.The extraction of the colour based features has mainly progressed in three main directions as selection of the colour space, quantization of the colour space and extraction of the colour feature. 


\subsection{Image Retrieval using Texture Content}

Like color, the texture is a powerful low-level feature for image search and retrieval applications. Much work has been done on texture analysis, classification, and segmentation for the last four decade, still there is a lot of potential for the research. So far, there is no unique definition for texture however, an encapsulating scientific definition as given in can be stated as Texture is an attribute representing the spatial arrangement of the grey levels of the pixels in a region or image.Texture is important component of human visual perception and can be effectively used for identifying different image regions. Compared with colour features, texture features indicate the macrostructure and microstructure of the images. [16]

\section{SUPPORT VECTOR MACHINE}

SVM is the state of art classification method which is introduced in 1992 by Bose, guy on and Vapnik. SVM is best tool for regression and classification [16]. Support vector machine may be defined as this is the linear function of the high dimensionality feature space which consist of the postulate space. SVM is a most beneficial technique for the data classification. Sometimes unsatisfied results are obtained using the neural networks and even it is easy to use. The classification task includes the training and testing data which consist the same data instances. Each sample in the training set consist the target values and its various attributes [7]. The major goal of SVM is to judge that the target value of various data instances in the testing set which are given only the attributes of the data. Classification in the SVM is the instance of supervised learning [ 12$]$.

\subsection{SVM Classification}

SVM is just like a NN. It works on feature vectors. In SVM the training and testing phase plays a very important role. By using SVM Classification we give training and them SVM gives the output as per training and gives output class-wise for example if we have selected a red rose as input query image them SVM classification firstly select all those images which have red colour that means all colour based feature can be retrieved using SVM classification [6][8][10].

\section{METHODOLOGY}

This section, explains the proposed method for Content based Image Retrieval system as follow:

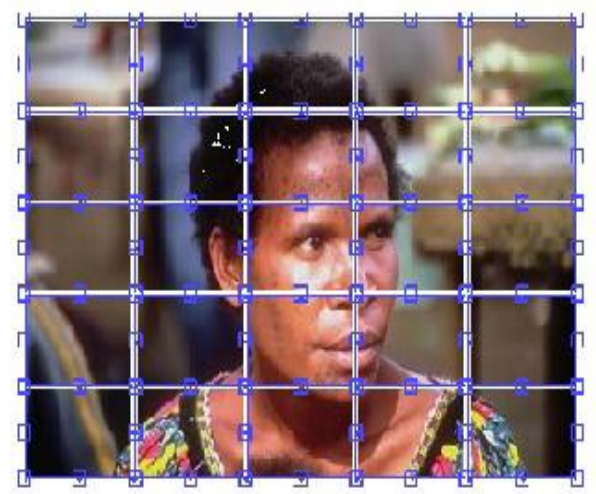

Figure2: Representation of image into $5 * 5$ rows and column

\subsection{Query Image}

Query Image is the desired image, which we want to retrieve from a large image collection. User can select any of the query image from a given images.

\subsection{Divide the imag}

Then we have divided the image into regions of 5 rows $* 5$. Columns of blocks of uniform size and assign fixed region value to each block that means image is divided into 25 block as shown in the figure 2 .

\subsection{Images Collection}

Images Collection includes different Wang database images which contain 1000 images. These images belong to different categories. Each category belongs to its specific class which is shown in below figure 3 .
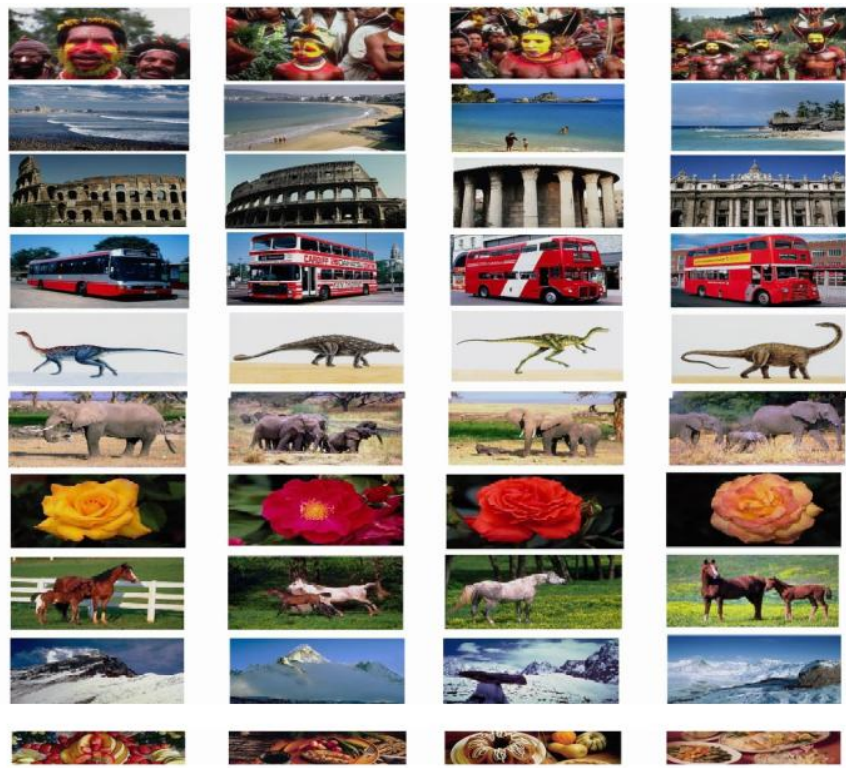

Figure 3: Sample of WANG image databas

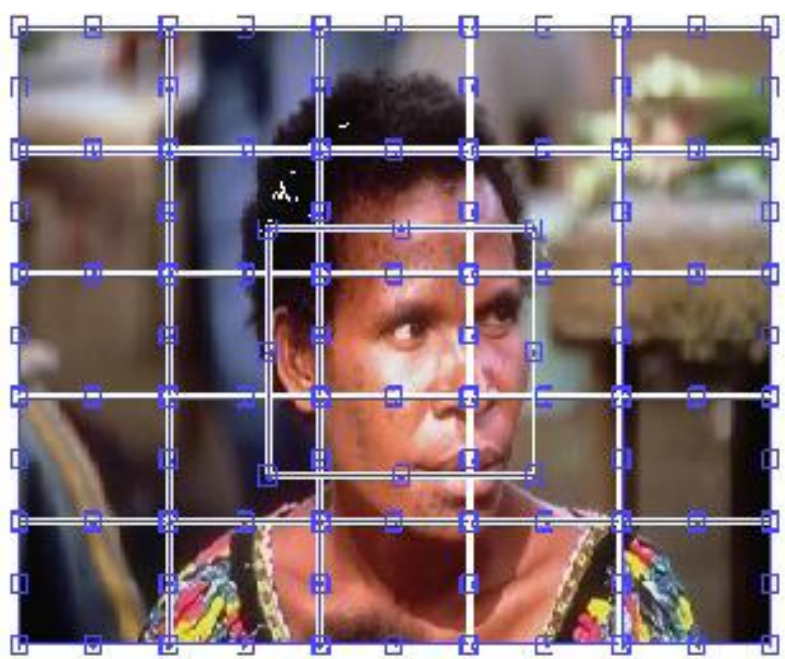

Figure 4: Selection of Region of interest into the image divided into $5 * 5$ rows and columns

\subsection{Selection of Region of Interest}

For the selection of region of interest we have taken the fixed window size $2 * 2$ on the image. Now those blocks we have checked that if the size of region is less than or equal to the size of block then it selects whole block under region of interest and 
if else it selects all the blocks under region of interest. The selection of ROI is shown in figure 4 .

\subsection{Similarity Measurement}

\subsubsection{Precision}

Precision measures the ability of the system to retrieve only models that are relevant .It can be seen as measure of exactness or quality.

Precision $P=\frac{T_{P}}{T_{P}+F_{P}}$

Where:-TP: - TRUE POSITIVE

FP: - FALSE POSITIVE

\subsubsection{Recall}

RECALL measures the ability of the system to retrieve all models that are relevant .It can be seen as the measure of completeness or quantity. Mathematically,

RECALL $R=\frac{T_{P}}{T_{P}+F_{N}}$

Where:-TP: - TRUE POSITIVE

FN: - FALSE NEGATIVE

\subsubsection{Accuracy}

Accuracy measures the how much exact output is retrieved by the system mathematically

Accuracy $=$ TP $\mathrm{TP}+\mathrm{TN}+\mathrm{FP}+\mathrm{FN}$

Where:-

TP: - TRUE POSITIVE

TN: - TRUE NEGATIVE

FP: - FALSE POSITIVE

FN: - FALSE NEGATIVE

\section{CONFUSION MATRIX}

Confusion matrix gives the information about actual and predicted classes. It gives the information of all true positive and true negative values, false positives and false negatives values in the form of matrix [15]. The diagonal elements of the confusion matrix gives the information about the all true positive rates. The parameters such as true positive rate, false positive rate, precision, recall and accuracy can be easily calculated from the confusion matrix. In this paper we have made confusion matrix at different training percent and then we have compare their values. The confusion matrixes are shown at the end of the paper.

\section{FLOW CHART OF PROPOSED SYSTEM}

The proposed system has been shown below in figure 5 .

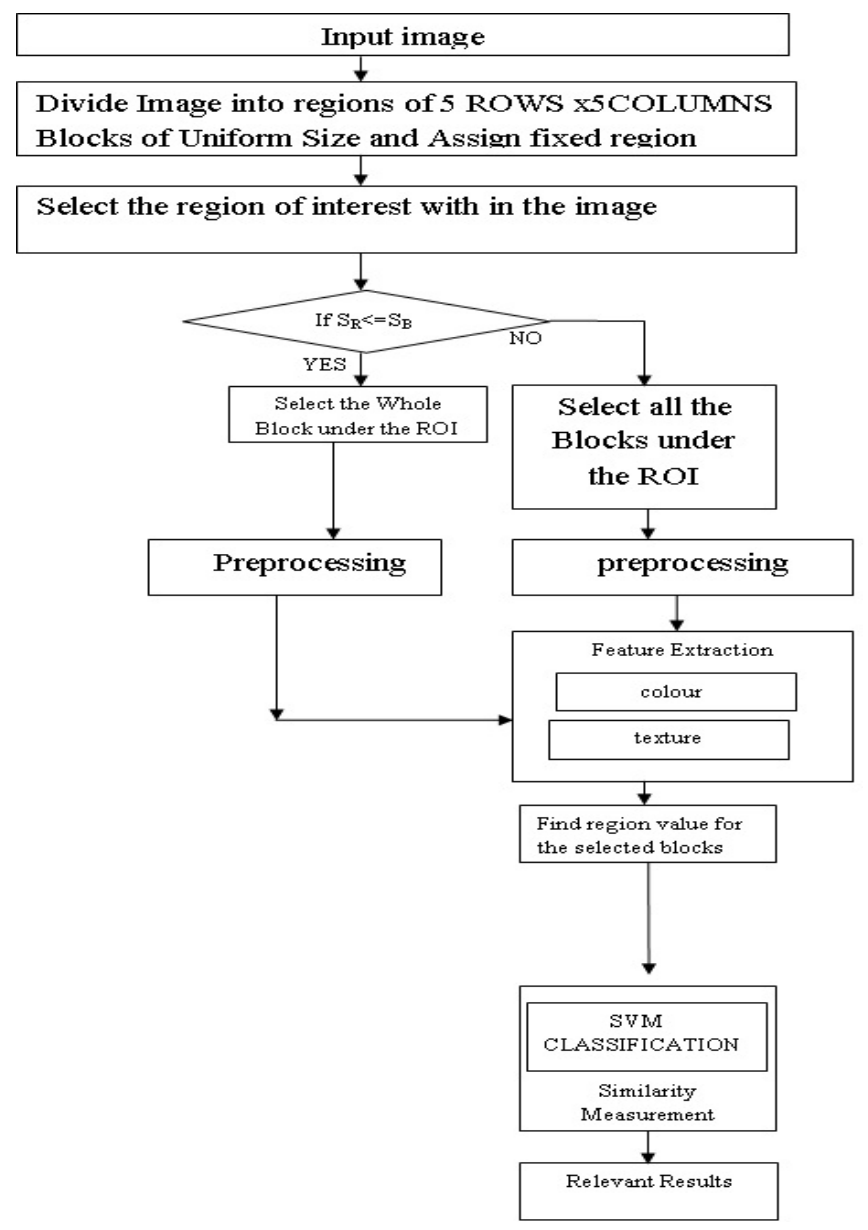

Figure 5:Proposed Flowchart of System

\section{RESULTS}

The present method has been implemented using MATLAB 2010.The system efficiency is evaluated by performing test on COREL database. Image database used in the experiment contains 1000 colored images of different categories. Texture features like Contrast, Mean, Standard Deviation, Energy and Entropy are extracted using GLCM and color features are extracted using color moment extracted are used for the image representation. A total of 50 query images are used for the system evaluation. For each query the top 35 retrieved images are displayed for feedback. When the query image is flower their retrieval is shown in figure7. When the query image is horse their retrieval is shown in figure8. When the query image is Diana sores their retrieval is shown in figure9.The system performance can be measured by evaluating precision $(\mathrm{P})$ and recall $(\mathrm{R})$ equations. The confusion matrix when SVM training percent is $20 \%, 50 \%, 80 \%$ respectively are shown in figure 10 , figure11 and figure 12. The accuracy shows that system gives better accuracy in their retrieval at different training and testing scenarios. The comparison graph is also shown in figure 6 . 
Table: 1 Comparison table of accuracy at different training percent and testing from whole database

\begin{tabular}{|c|c|c|}
\hline $\mathbf{2 0 \%}$ & $\mathbf{5 0 \%}$ & $\mathbf{8 0 \%}$ \\
\hline $76.55 \%$ & $84.28 \%$ & $85.37 \%$ \\
\hline $83.82 \%$ & $85.99 \%$ & $86.37 \%$ \\
\hline $78.96 \%$ & $80.83 \%$ & $83.12 \%$ \\
\hline $85.94 \%$ & $90.58 \%$ & $92.18 \%$ \\
\hline $97.97 \%$ & $99.25 \%$ & $99.53 \%$ \\
\hline $79.82 \%$ & $86.29 \%$ & $87.22 \%$ \\
\hline $93.23 \%$ & $95.72 \%$ & $97.10 \%$ \\
\hline $88.14 \%$ & $91.83 \%$ & $95.34 \%$ \\
\hline $85.09 \%$ & $88.01 \%$ & $89.21 \%$ \\
\hline $85.47 \%$ & $87.94 \%$ & $89.79 \%$ \\
\hline
\end{tabular}

\section{ComparisonAccuracy Graph at Different Trainning Percent}

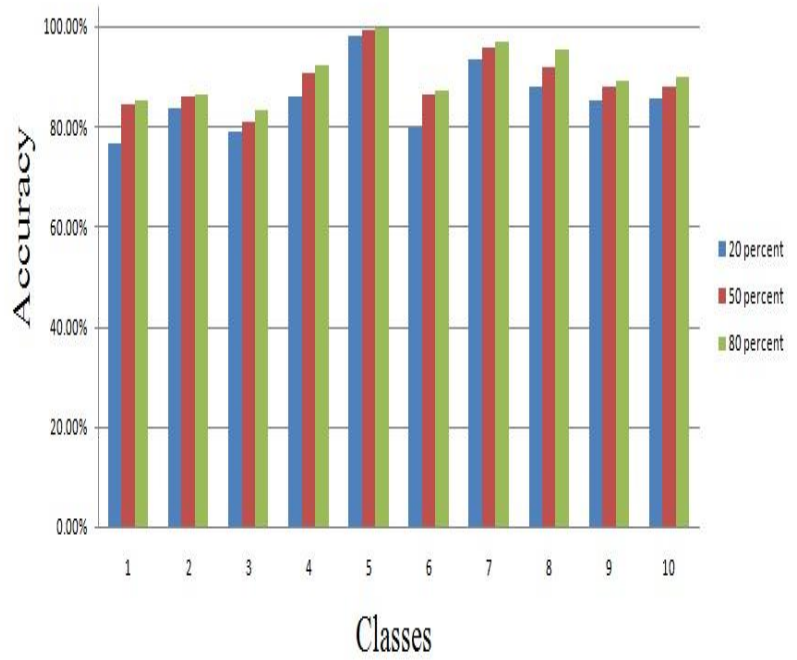

Figure6: Comparison accuracy graph at different training percent

\section{CONCLUSION}

In this study, a selective region based system is proposed for content based image retrieval (CBIR) system. In the proposed method image texture and color features are utilized along with

ROI. The relevant images are retrieved from large database by comparing the query image and images in database using city block distance. The SVM classification is used to retrieve the relevant images from a large database. Accuracy is evaluated by using SVM classification and the confusion matrix is drawn to conclude our results. The proposed method is simple and fast in retrieval The average accuracy evaluated for the proposed approach is $90 \%$.

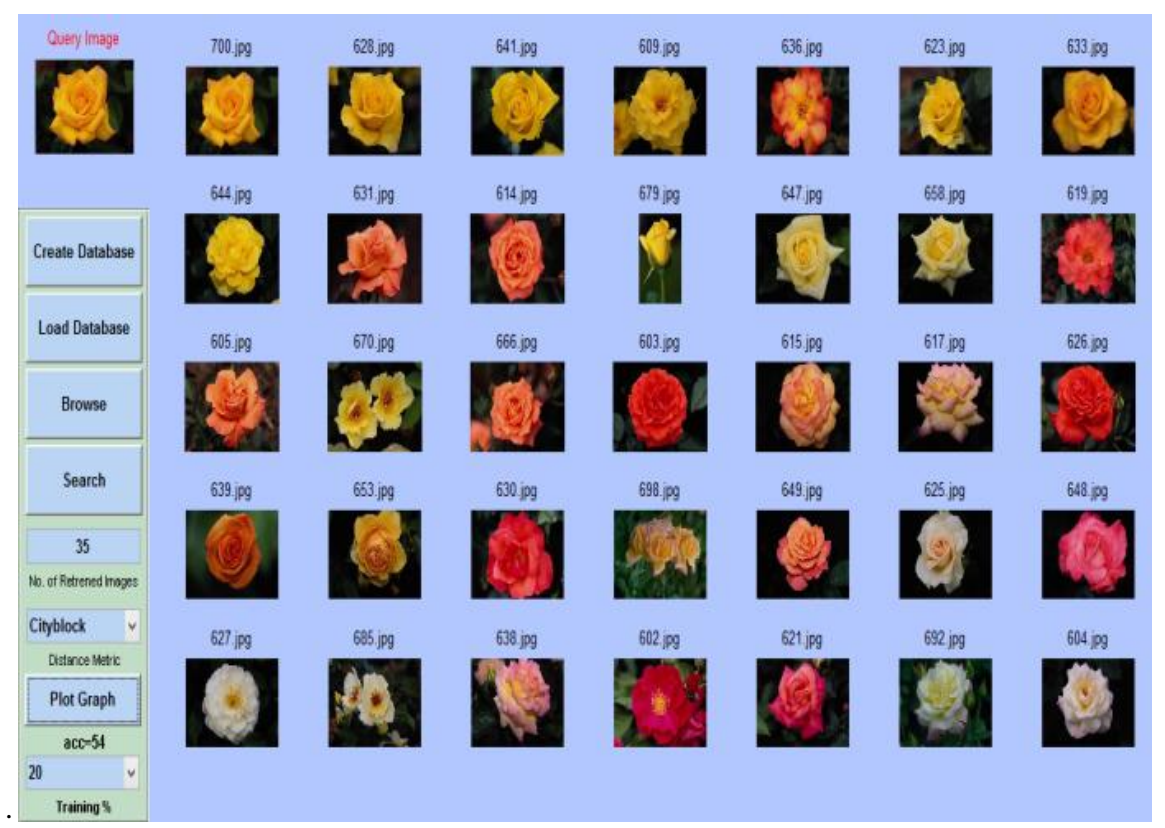

Figure7: Retrieval of images from the class of flower 


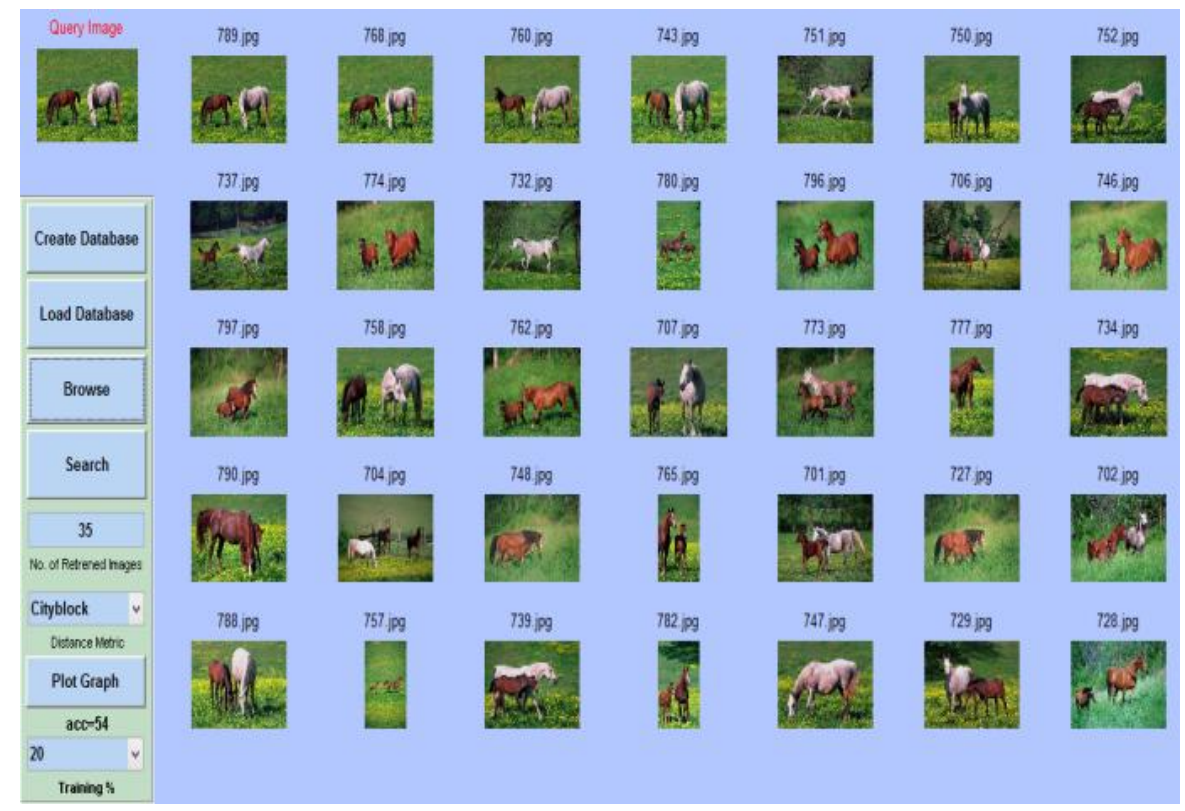

Figure8: Retrieval of Images from the Class of Horse

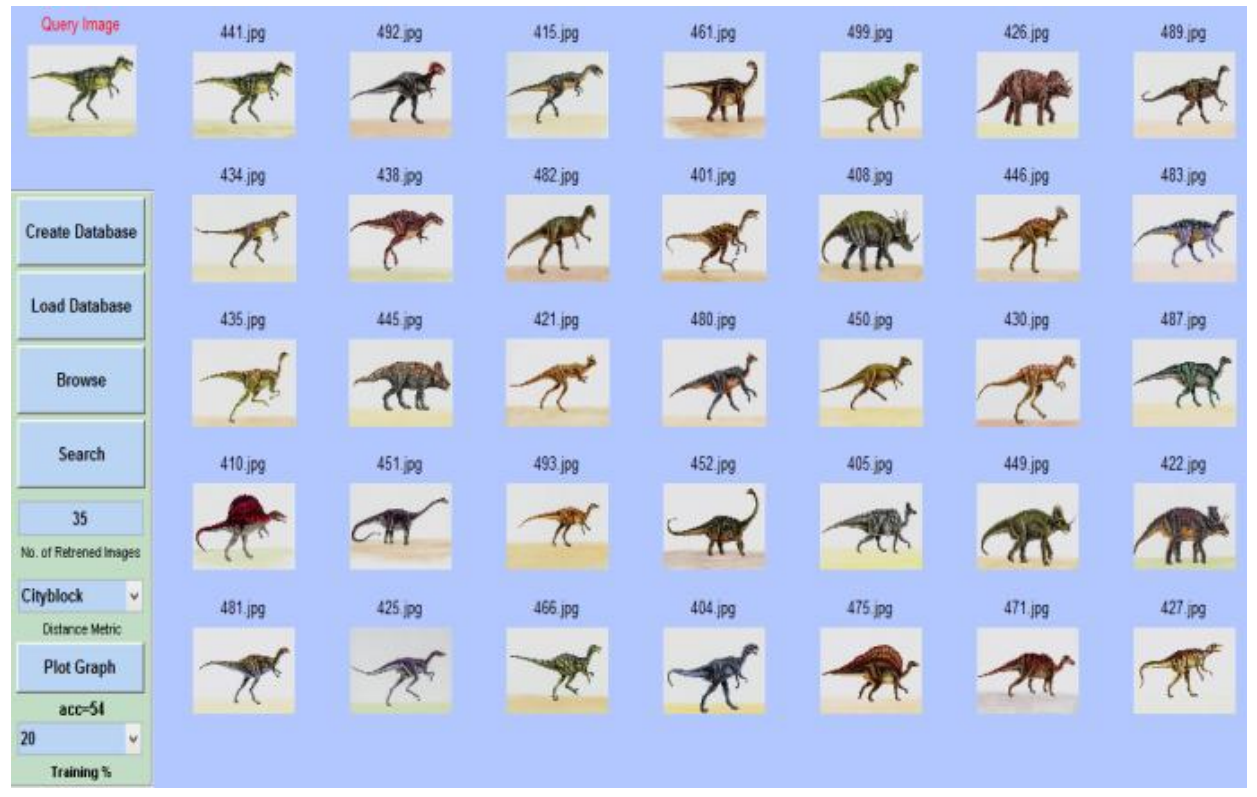

Figure9:- Retrieval of Images from the Class of Diana sores

\section{ACKNOWLEDGMENT}

Our thanks to the experts and specially my guide and all my worthy friends who gave their contribution in making this work.

\section{REFERENCES}

[1] N. Shrivastava and V. Tyagi, "Content based image retrieval based on relative locations of multiple regions of interest using selective regions matching," Inf. Sci. (Ny)., vol. 259, pp. 212-224, Feb. 2014.

[2] Sasheendran, Nivya, and C. Bhuvaneswari. "An effective CBIR (Content Based Image Retrieval) approach using Ripplet transforms." Circuits, Power and Computing Technologies (ICCPCT), 2013 International Conference on. IEEE, 2013.

[3] Singha, Manimala, and K. Hemachandran. "Content based image retrieval using color and texture." Signal \& Image
Processing: An International Journal (SIPIJ) 3.1 (2012): 39-57.

[4] Baharudin, Baharum. "Effective content-based image retrieval: Combination of quantized histogram texture features in the DCT domain." Computer \& Information Science (ICCIS), 2012 International Conference on. Vol. 1. IEEE, 2012.

[5] Zhang, Lei, Fuzong Lin, and Bo Zhang. "Support vector machine learning for image retrieval." Image Processing, 2001. Proceedings. 2001 International Conference on. Vol. 2. IEEE, 2001

[6] Chen, Yunqiang, Xiang Sean Zhou, and Thomas S. Huang. "One-class SVM for learning in image retrieval." Image Processing, 2001. Proceedings. 2001 International Conference on. Vol. 1. IEEE, 2001.

[7] Jain, Sonali, and Satyam Shrivastava. "A novel approach for image classification in Content based image retrieval 
using support vector machine." Intermational Journal of Computer Science \& Engineering (IJCSET) Vol 4 (2013).

[8] Prabhu, Jeyanthi, and Jawahar Senthil Kumar. "Wavelet Based Content Based Image Retrieval Using Color and Texture Feature Extraction bY Gray Level Coocurence Matrix and Color Coocurence Matrix." Journal of Computer Science 10.1 (2014): 15.

[9] Kumar, K. Ashok, and YV Bhaskar Reddy. "Content Based Image Retrieval Using SVM Algorithm." international Journal of Electrical and Electronics Engineering (IJEEE) ISSN (PRINT) 2231 (2012): 5284.

[10] Huang, Zhi-Chun, et al. "Content-based image retrieval using color moment and Gabor texture feature." Machine Learning and Cybernetics (ICMLC), 2010 International Conference on. Vol. 2. IEEE, 2010.

[11] Iqbal, Kashif, Michael O. Odetayo, and Anne James. "Content-based image retrieval approach for biometric security using colour, texture and shape features controlled by fuzzy heuristics." Journal of Computer and System Sciences 78.4 (2012): 1258-1277.

[12] Kaur, Simardeep, and Dr Vijay Kumar Banga. "Content based image retrieval: Survey and comparison between rgb and hsv model." International Journal of Engineering Trends and Technology 4.4 (2013): 575-579.

[13] Wang, Xingyuan, and Zongyu Wang. "The method for image retrieval based on multi-factors correlation utilizing block truncation coding." Pattern Recognition 47.10 (2014): 3293-3303.

[14] Penatti, Otávio AB, Eduardo Valle, and Ricardo da S Torres. "Comparative study of global color and texture descriptors for web image retrieval." Journal of Visual Communication and Image Representation 23.2 (2012): 359-380.

[15] Bhavneet Kaur, Sonika Jindal, "Accelerating CBIR System using Graphics Processing Unit in OPEN CV environment", (IJCA) International Journal of Computer Applications, Volume 8,Page No. 8,September,(2015) ISSN: $0975-8887$

[16] Bhavneet Kaur, Sonika Jindal, "An Implementation of Feature Extraction over medical Images on OPEN CV Environment", (ICDCCom)2014 International Conference on Devices, Circuits and Communications, Pages $\quad 1-6, \quad$ September 2014,DOI: 10.1109/ICDCCom.2014.7024695

\section{APPENDIX}

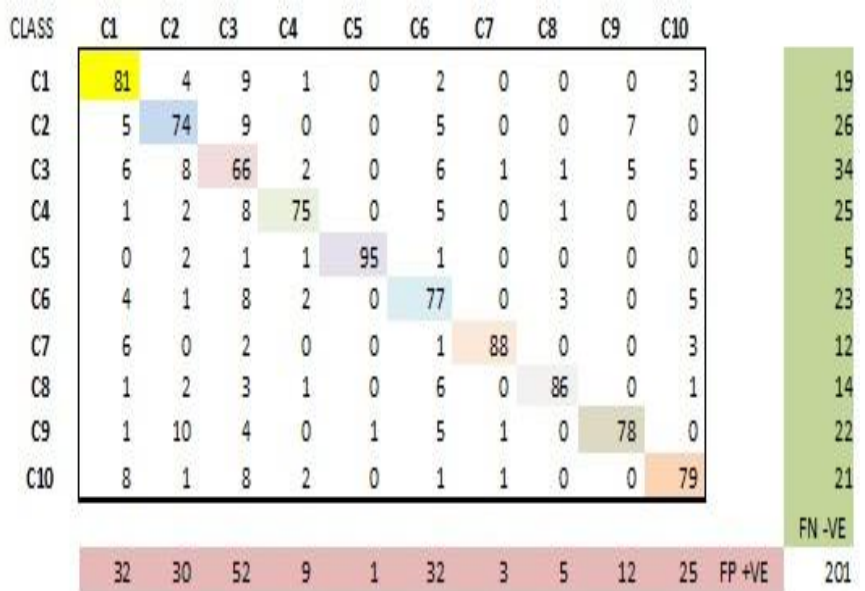

Figure 10.Confusion matrix when svm trainning is $20 \%$

\begin{tabular}{|c|c|c|c|c|c|c|c|c|c|c|c|c|}
\hline CLASS & $\mathrm{Cl}$ & $\mathrm{C} 2$ & $\mathrm{C} 3$ & $\mathrm{CH}$ & $\mathrm{C5}$ & $C 6$ & $\mathrm{C7}$ & $C 8$ & $\mathrm{Cg}$ & $\mathrm{C} 10$ & & \\
\hline $\mathrm{Cl}$ & 79 & 4 & 3 & 0 & 0 & 3 & 0 & 0 & 1 & 10 & & 21 \\
\hline$Q$ & 6 & 64 & 8 & 0 & 0 & 9 & 0 & 2 & 7 & 4 & & 36 \\
\hline C3 & 21 & 7 & 45 & 1 & 0 & 6 & 2 & 2 & 4 & 12 & & 55 \\
\hline C4 & 19 & 1 & 14 & 37 & 0 & 8 & 1 & 4 & 3 & 13 & & 63 \\
\hline C5 & 1 & 4 & 0 & 0 & 92 & 0 & 0 & 0 & 1 & 2 & & 8 \\
\hline 06 & 11 & 5 & 7 & 0 & 0 & 60 & 1 & 4 & 6 & 6 & & 40 \\
\hline C7 & 9 & 0 & 1 & 0 & 0 & 2 & 83 & 1 & 0 & 4 & & 17 \\
\hline C8 & 21 & 2 & 7 & 0 & 0 & 19 & 0 & 50 & 0 & 1 & & 50 \\
\hline$c 9$ & 14 & 22 & 9 & 0 & 0 & 8 & 0 & 1 & 45 & 1 & & 55 \\
\hline \multirow[t]{3}{*}{$\mathrm{C} 10$} & 23 & 4 & 1 & 1 & 0 & 5 & 4 & 2 & 1 & 59 & & 41 \\
\hline & & & & & & & & & & & & FN-VE \\
\hline & 125 & 49 & 50 & 2 & 0 & 60 & 8 & 16 & 23 & 53 & $F P+V E$ & 386 \\
\hline
\end{tabular}

Figure 11.Confusion matrix when svm trainning is $\mathbf{5 0 \%}$

\begin{tabular}{|c|c|c|c|c|c|c|c|c|c|c|c|c|}
\hline CLASS & $\mathrm{Cl}$ & $\mathrm{C} 2$ & C3 & $\mathrm{CH}$ & $\mathrm{C5}$ & $C 6$ & C7 & $\mathrm{CB}$ & $C 9$ & $\mathrm{C10}$ & & \\
\hline $\mathrm{Cl}$ & 93 & 2 & 5 & 0 & 0 & 0 & 0 & 0 & 0 & 0 & & 7 \\
\hline $\mathrm{Cl}$ & 2 & 89 & 2 & 0 & 0 & 3 & 0 & 0 & 3 & 1 & & 11 \\
\hline C3 & 4 & 1 & 90 & 2 & 0 & 2 & 0 & 0 & 0 & 1 & & 10 \\
\hline $\mathrm{CA}$ & 1 & 1 & 1 & 93 & 0 & 0 & 0 & 2 & 1 & 1 & & 7 \\
\hline$C 5$ & 0 & 0 & 0 & 0 & 100 & 0 & 0 & 0 & 0 & 0 & & 0 \\
\hline C6 & 2 & 1 & 0 & 1 & 0 & 94 & 0 & 1 & 0 & 1 & & 6 \\
\hline C7 & 1 & 0 & 0 & 0 & 0 & 0 & 95 & 0 & 0 & 4 & & 5 \\
\hline$C 8$ & 2 & 2 & 1 & 0 & 0 & 1 & 0 & 94 & 0 & 0 & & 6 \\
\hline $\mathrm{Cg}$ & 1 & 4 & 3 & 0 & 0 & 1 & 0 & 1 & 90 & 0 & & 10 \\
\hline $\mathrm{C} 10$ & 3 & 1 & 4 & 0 & 0 & 0 & 1 & 0 & 0 & 91 & & 9 \\
\hline
\end{tabular}

Figure 12.Confusion matrix when svm trainning is $80 \%$ 